Conference Paper

\title{
Analysis of the effects on the pitching, rolling and yawing rate of a v- tail configured quadcopter
}

McClanahan, C., Bolam, R., Vagapov, Y. and Anuchin, A.

This is a paper presented at the 25th Int. Workshop on Electric Drives: Optimization in Control of Electric Drives, Moscow, Russia

Copyright of the author(s). Reproduced here with their permission and the permission of the conference organisers.

\section{Recommended citation:}

McClanahan, C., Bolam, R., Vagapov, Y. and Anuchin, A. (2019). Analysis of the effects on the pitching, rolling and yawing rate of a v-tail configured quadcopter. In: Proc. 25th Int. Workshop on Electric Drives: Optimization in Control of Electric Drives, Moscow, Russia, 30 January - 2 February 2019, pp. $1-7$. 


\section{Analysis of the Effects on the Pitching, Rolling and Yawing Rate of a V-tail Configured Quadcopter}

\author{
Christopher McClanahan \\ Glyndwr University \\ Wrexham, UK \\ Yuriy Vagapov \\ Glyndwr University \\ Wrexham, UK
}

\author{
Robert Cameron Bolam \\ Glyndwr University \\ Wrexham, UK \\ Alecksey Anuchin \\ Moscow Power Engineering Institute \\ Moscow, Russia
}

\begin{abstract}
Many different projects have been focused on multirotor aircraft, especially on quadcopters, but there are only a few papers relating to the dynamic effects on quadcopters with tilted motors. In this paper, a quadcopter has been modelled to allow flight simulation under differing motor tilt angle configurations. The simulation has also been validated by building a quadcopter of known physical attributes and with on-board instrumentation and telemetry to $\log$ its attitude and motor control inputs (PWM signals). The conversion from a PWM signal to the rpm was achieved by determining the relationship between the PWM signal and the thrust generated. Both of these parameters being recorded on a test bench. Thrust is calculated for each motor individually, since the angled motors generate different advance ratios and hence different thrust coefficients. The main focus of this research was on the effect of the different coefficients and the thrust components acting in the $x-y$ plane of the quadcopter body frame.
\end{abstract}

Keywords-drones, unmanned aircraft system, quadcopter, $V$-tile configuration

\section{INTRODUCTION}

Over the past few years the fastest growing sector of the aerospace industry has arguably been that of unmanned and autonomous air vehicles, more popularly known as "drone" technology.

In the United States the Federal Aviation Administration (FAA) have predicted Unmanned Aircraft Systems (UAS) growth for 3.5 million hobbyist and 442,000 commercial aircraft to be operational by 2021 [1]. Similarly, the importance of the emerging drone market has been widely recognised in Europe where there are at least 2495 operators and 114 manufacturers of drones up to $150 \mathrm{~kg}$ MTOM [2]. Nowadays drones have already established their usefulness in a variety of industries, such as the Media and Emergency Services and indeed their deployment for surveying missions in support of farming, archaeology and civil engineering activities is a commonplace occurrence [3]. More recent technological developments for drones have been made in areas such as formation "swarm" flights and "sense and avoid" technology for multirotor craft [4].

Developing drone control theory and the creation of accurate theoretical models is an important step towards understanding and improving drone agility and control. Numerous studies have been made on the control of multirotor aircraft resulting in proposals with differing approaches and improvements of their control. For example, studies have been carried out on the control characteristics of a multirotor aircraft landing autonomously on a moving platform [5]. Likewise, Bergamasco and Lovera [6] have identified linear models for the dynamics of a hovering Quadrotor and Omari et al. [7] have proposed novel hardware and software architectures to achieve the nonlinear control of multirotor helicopters.

Studies have also been conducted to improve the survivability and safety of a multirotor after being subjected to an impact. This has resulted in the emergence of different conceptual approaches. One concept [8] uses the deformation of a specially designed multirotor frame to absorb the impact energy; whilst another concept avoids the loss of control with a post-impact recovery controller. The latter study [9] also refers to an area for future work once a model of the impact of a quadrotor on a wall has been developed.

In order to develop a multirotor aircraft, which utilises vector controlled thrust, the effects of tilt on different motor configurations must be understood [10]. However, there is only a limited amount of literature available in this field of study although, Efraim at al. [11] have proposed a theoretical model for a Quadcopter with motors in a dihedral configuration and also some related analytical experiments have been performed of a V-tail quad-rotor dynamics [12]. The purpose of this work is to present the combined theoretical and empirical results of a dynamic analysis of the effects on the pitching, rolling and yawing rate of a $\mathrm{V}$-tail configured quadcopter drone.

\section{V-TAIL QUADCOPTER MATHEMATICAL MODELLING}

The main difference between a $\mathrm{V}$-tail configuration quadcopter and a more conventional $\mathrm{H}$ or $\mathrm{X}$ type quadcopter is that the inclined tail motors of the V-tail should provide faster yaw control. Improving a quadcopter's manoeuvrability in this way should benefit high speed quadcopter deployments within obstacle congested environments such as those encountered during racing, indoor flights or through undergrowth and forested regions. However, it is anticipated that this perceived advantage may come at the price to the quadcopter's overall lifting performance and pitch and roll response. To investigate these effects, the following mathematical model was constructed.

In this work a multirotor aircraft with no tilted motors is referred to as a "normal configuration", where the 


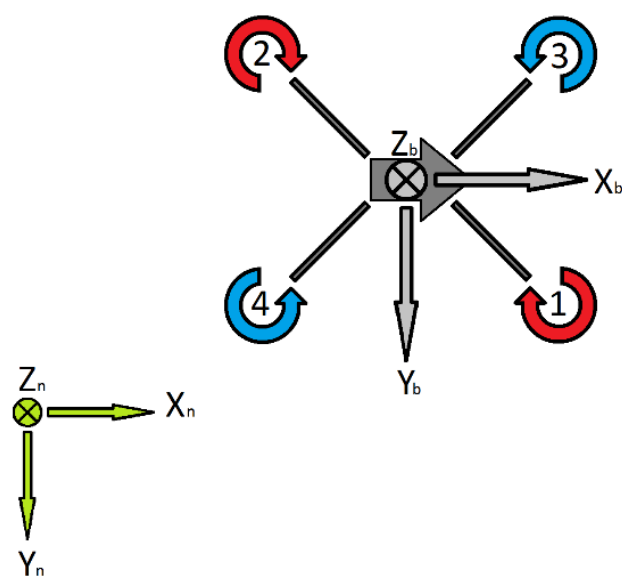

Fig. 1. Navigation frame and body frame, with the motor positions and direction of rotation.

rotational axis of each motor is parallel to $Z_{b}$ axis. In the "dihedral configuration", all motors are equally tilted around $Z_{b}$ and $Y_{b}$ axis towards the centre of gravity (14 deg.), other than in the $\mathrm{V}$-tail configuration, where the rear motors (M2 and M4) are tilted around $X_{b}$ axis towards each other (30 deg.).

To model a multirotor aircraft it is seen as a rigid body, moving in the coordination of the navigation frame with the index $n$ (Fig. 1). Due to the small distances travelled by the multirotor aircraft, earth's curvature is neglected [11]. The coordination system of the object is called body frame with the index $b$, in this case the Quadcopter (Fig. 1).

The conversion of different force vectors between navigation frame and body frame, Euler angles and rotation matrices are used as following.

$$
\begin{aligned}
\mathbf{R}_{\sigma x}(\phi) & =\left[\begin{array}{ccc}
1 & 0 & 0 \\
0 & \cos \phi & \sin \phi \\
0 & -\sin \phi & \cos \phi
\end{array}\right] \\
\mathbf{R}_{\sigma y}(\theta) & =\left[\begin{array}{ccc}
\cos \theta & 0 & -\sin \theta \\
0 & 1 & 0 \\
\sin \theta & 0 & \cos \theta
\end{array}\right] \\
\mathbf{R}_{\sigma z}(\psi)= & {\left[\begin{array}{ccc}
\cos \psi & \sin \psi & 0 \\
-\sin \psi & \cos \psi & 0 \\
0 & 0 & 1
\end{array}\right] }
\end{aligned}
$$

where $\phi$ is Euler angle around $X[\mathrm{rad}] ; \theta$ is Euler angle around $Y[\mathrm{rad}] ; \psi$ is Euler angle around $Z$ [rad].

Since the body frame is usually tilting in 3 directions at the same time a rotation matrix for all axes is needed using the rotation sequence roll $\phi$, pitch $\theta$, yaw $\psi$ [9], [13], [14].

$$
\mathbf{C}_{b}^{n}=\mathbf{R}_{\sigma x}(\phi) \times \mathbf{R}_{\sigma y}(\theta) \times \mathbf{R}_{\sigma z}(\psi)
$$

where $\mathbf{C}_{b}^{n}$ is rotation matrix body to navigation frame (3).

If the conversion needs to be done from the navigation frame to the body frame the transposed rotation matrix is used as following.

$$
\begin{aligned}
\mathbf{C}_{n}^{b} & =\mathbf{C}_{b}^{n T} \\
\mathbf{C}_{b}^{n} & =\left[\begin{array}{c}
\cos \theta \cos \psi \\
\sin \phi \sin \theta \cos \psi-\cos \phi \sin \psi \\
\sin \phi \sin \psi+\cos \phi \sin \theta \cos \psi
\end{array}\right.
\end{aligned}
$$

where $\mathbf{C}_{n}^{b}$ is rotation matrix navigation to body frame.

The relation of Newton's second law between forces acting on a rigid body, its mass and acceleration is the basis of the model.

$$
\ddot{r} \times m=\bar{F}_{b g}-\bar{F}_{b t}+\bar{F}_{\text {disturbance }}+\bar{F}_{d r a g}
$$

where $\ddot{r}$ is acceleration of the multirotor aircraft $\left[\mathrm{m} / \mathrm{s}^{2}\right] ; m$ is mass of the multirotor aircraft $[\mathrm{kg}] ; \bar{F}_{b g}$ is force vector of gravity $[\mathrm{N}] ; \bar{F}_{b t}$ is thrust force vector [N]; $\bar{F}_{\text {disturbance }}$ is disturbance vector $[\mathrm{N}] ; \bar{F}_{d r a g}$ drag vector $[\mathrm{N}]$.

Gravity acts along the $Z_{n}$ axis and is converted to the body frame with the transposed rotation matrix from (3).

$$
\bar{F}_{b g}=m_{t o t} \times \mathbf{C}_{n}^{b} \times\left[\begin{array}{l}
0 \\
0 \\
g
\end{array}\right]
$$

where $g$ is acceleration due to gravity $\left[\mathrm{m} / \mathrm{s}^{2}\right]$

Since the propeller data of the UIUC Propeller Database of [15] (APC9x4.5MR, APC9x4.5MRP) is used for the thrust calculations, the equations $(7)-(11)$ are provided by this database. To consider the different velocities of the airstream normal to the propeller disc, all the calculations concerning the propellers are done individually for each motor, in order to get different propeller coefficient for the different motors. Blade flapping described in [7], [14], [16] is neglected, since only small UAV velocities are simulated.

$$
J=\frac{V_{b p}}{n \times D}
$$

where $J$ is advance ratio; $V$ is velocity of air (wind tunnel) $[\mathrm{m} / \mathrm{s}] ; D$ is propeller diameter $[\mathrm{m}] ; n$ is propeller speed $[\mathrm{rev} / \mathrm{s}]$.

$$
C_{T}=\frac{F_{b t}}{\rho \times n^{2} \times D^{4}}
$$

where $C_{T}$ is thrust coefficient; $\rho$ is air density $\left[\mathrm{kg} / \mathrm{m}^{3}\right]$.

$$
C_{P}=\frac{P}{\rho \times n^{3} \times D^{5}}
$$

where $C_{P}$ is power coefficient

$$
C_{q}=\frac{Q}{\rho \times n^{2} \times D^{5}}
$$

where $C_{q}$ is torque coefficient.

$$
\eta=J \times \frac{C_{T}}{C_{P}}
$$

where $\eta$ is propeller efficiency.

In order to calculate the advance ratio $J(7)$, the velocity of the airstream normal to the propeller disc is calculated using (12). The part of this equation under the square root is derived from calculating the height of a pyramid, from the length of the side edge, with the side angles given.

$$
V_{b p}=\dot{X}_{b} \sin \beta_{y}+\dot{Y}_{b} \sin \beta_{x}+\sqrt{\frac{\dot{Z}_{b}^{2}}{1+\tan \beta_{x}^{2}+\tan \beta_{y}^{2}}}
$$

$$
\left.\begin{array}{cc}
\cos \theta \sin \psi & -\sin \theta \\
\cos \phi \cos \psi+\sin \phi \sin \theta \sin \psi & \sin \phi \cos \theta \\
\cos \phi \sin \theta \sin \psi-\sin \phi \cos \psi & \cos \phi \cos \theta
\end{array}\right]
$$


The constant $T_{q}$ (Force to Torque relation) has been redefined with respect to [15] since this data is used for thrust and torque calculations. Rearranging thrust gives

$$
\frac{F_{b t}}{C_{T}}=\rho \times n^{2} \times D^{4}
$$

and rearranging torque gives

$$
\frac{Q}{C_{q} \times D}=\rho \times n^{2} \times D^{4}
$$

The relation between the power coefficient and the torque coefficient is

$$
C_{q}=\frac{C_{P}}{2 \pi}
$$

Equate thrust and torque, insert the power coefficient from above and rearrange gives

$$
Q=F \times\left[\frac{C_{p}}{2 \pi} \times D \times C_{t}^{-1}\right]
$$
[15].

The part in the brackets (16) is $T_{q}$ defined as following

$$
T_{q}=\left[\frac{C_{p}}{2 \pi} \times D \times C_{t}^{-1}\right]=\frac{Q}{F}
$$

In [9], [14], [16], [17], the main thrust acts in parallel, but tilting the motors means splitting the forces into the $X_{b}$, $Y_{b}$ and $Z_{b}$ components. This is achieved with (18), which has the same roots as (12).

$$
\begin{gathered}
F_{b t z}=-\sqrt{\frac{F_{b t}^{2}}{1+\tan \beta_{x}^{2}+\tan \beta_{y}^{2}}} \\
\bar{F}_{b t m(i)}=\left[\begin{array}{c}
F_{b t z} \tan \beta_{y} \\
F_{b t z} \tan \beta_{x} \\
F_{b t z}
\end{array}\right]=\left[\begin{array}{c}
F_{b t x} \\
F_{b t y} \\
F_{b t z}
\end{array}\right]
\end{gathered}
$$

(19) represents thrust force converted into the body frame, where $i$ is the motor number. Therefore, the following (20) is summing the motor forces together. and

$$
\bar{F}_{b t}=\sum_{i=1}^{4} \bar{F}_{b t m(i)}
$$

Disturbance force $\bar{F}_{\text {disturbance }}$ is used to induce a disturbance in the simulation, whereas drag force $\bar{F}_{\text {drag }}$ is due to the high expenditure of time for the implementation neglected.

The quadcopter is affected by torque caused by thrust $\bar{M}_{b \text { thrust }}$ and gyroscopic forces of the motor $\bar{M}_{b}$ [9]. Against this acts the inertia of the body and the centripetal force as following.

$$
\bar{M}_{b}=\mathbf{I} \times \overline{\ddot{\sigma}}+\overline{\dot{\sigma}} \times(\mathbf{I} \times \overline{\dot{\sigma}})
$$

where $\mathbf{I}$ is matrix of inertia $\left[\mathrm{kgm}^{2}\right]$

The Eigenvalues of the matrix of inertia are used and the matrix of inertia is described as:

$$
\mathbf{I}=\left[\begin{array}{ccc}
I_{x x} & 0 & 0 \\
0 & I_{y y} & 0 \\
0 & 0 & I_{z z}
\end{array}\right]
$$

Similar to the thrust force, the moments of the motors act around every axis after tilting the motors. (23) describes the moment caused by thrust and drag of the propeller on an around two axis tilted motor.

$$
\bar{M}_{b t m(i)}=\left[\begin{array}{l}
F_{y} l_{z}+F_{z} l_{y}+F_{x} T_{q} \\
F_{x} l_{z}+F_{z} l_{x}+F_{y} T_{q} \\
F_{y} l_{x}-F_{x} l_{y}+F_{z} T_{q}
\end{array}\right]
$$

where $l_{(x ; y ; z)}$ is distance from the force vector to the centre of gravity $[\mathrm{m}]$.

To calculate the gyroscopic moment around every axis, the angular velocity of the motor must be split into the components acting in the body frame.

$$
\omega_{\text {motz }}=\sqrt{\frac{\omega_{m o t}^{2}}{1+\tan \beta_{x}^{2}+\tan \beta_{y}^{2}}}
$$

Angular velocity of the tilted motor along every axis is represented by the following, where $i$ is the motor number.

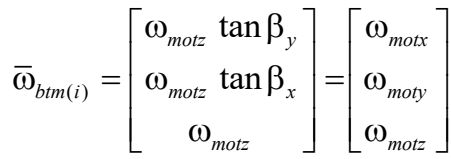

Therefore, the gyroscopic moment acting on one tilted motor along the axis of the body frame can be found from the following.

$$
M_{b g m(i)}=\left[\begin{array}{c}
\dot{\sigma}_{y} \omega_{m o t z}+\dot{\sigma}_{z} \omega_{m o t y} \\
\dot{\sigma}_{x} \omega_{m o t z}+\dot{\sigma}_{z} \omega_{m o t x} \\
\dot{\sigma}_{y} \omega_{m o t x}+\dot{\sigma}_{x} \omega_{m o t y}
\end{array}\right] \times I_{m o t}
$$

After calculating every moment caused by each individual motor, they are added to summarise the motor moments together.

$$
\bar{M}_{b}=\sum_{i=1}^{4}\left[M_{b t m(i)}+M_{b g m(i)}\right]
$$

\section{PRACTICAL EXPERIMENT TO VALIDATE MATHEMATICAL MODEL}

As a validation test, a pivot mechanism where the motor is mounted on the standing lever pointing forwards has been used. A weighing module with an accuracy of a 10th of a gram is mounted on the other side of the lever. The pivot arm is preloaded against the scale with an elastic band, to ensure accurate measurements during test with little thrust and to prevent uncontrolled movements during sudden thrust drops (Fig. 2). The pivot axis is located in the middle of the lever; thus the scale displays the actual thrust of the motor after correct zeroing.

To minimize the risk of compromising the results by using different hardware, the multirotor aircraft used in the test flights has been also used to control the motor in the test bench, which is taken from the UAV itself.

The test itself consisted of three test runs, which were averaged to reduce the impact of single outliers. Each cycle consisted of nine thrust tests. The throttle was controlled via the ground station, starting at $10 \%$ and increasing in $10 \%$ steps at each run, until $100 \%$ was reached.

In order to verify the mathematical model the rotational speed of the motors during the test flights must be known. Since the recording of the actual $\mathrm{rpm}$ of the motors involves a high degree of instrumentation complexity, the PWM signal to the ESCs is recorded instead using a data 


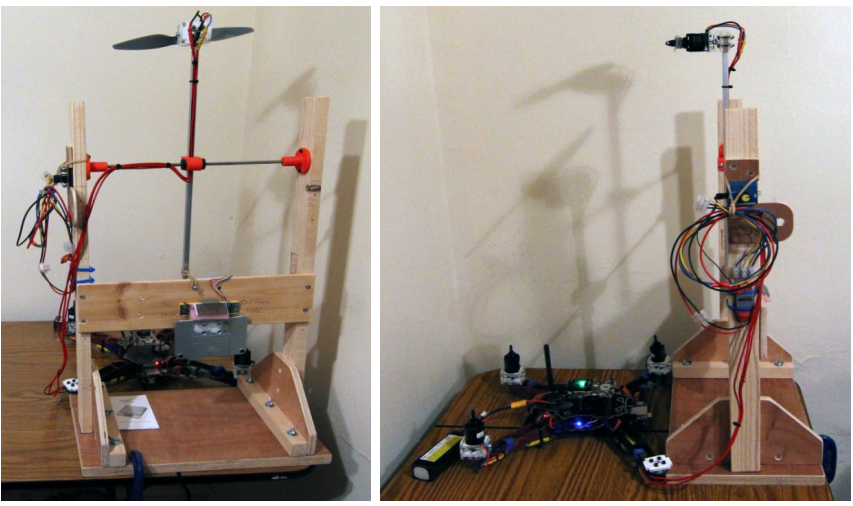

Fig. 2. The test setup

logger. The data logger, which is based on an Arduino, did not delay or interfere with the motor the PWM signals. Using this data it was possible to use an existing thrust test stand and calculate the actual rpm for a given propeller thrust data. The firmware used is a slightly modified version of the data logging firmware provided by Adafruit [18].

The verification consisted of loading the motor data into the theoretical model, running the simulation and comparing it with the actual attitude of the UAV. Since the theoretical model of the UAV does not include any tolerances and inaccuracies, some adjustments were required in order to match the real model. The lengths of the arms have been adjusted in the range of a few millimetres and the rpm of all four motors was reduced to $70 \%$ and the rpm of motor 2 increased by $4.5 \%$. It is assumed that this individual discrepancy is caused by an unsymmetrical ESC calibration. The overall reduction of the motor rpm is contributed to the wind and drag, which was not considered during the test.

The divergence of the validation, shown in Fig. 3, reveals a maximum discrepancy of $0.315 \mathrm{rad}$ of the roll angle. It was also assumed, that the huge divergence of the
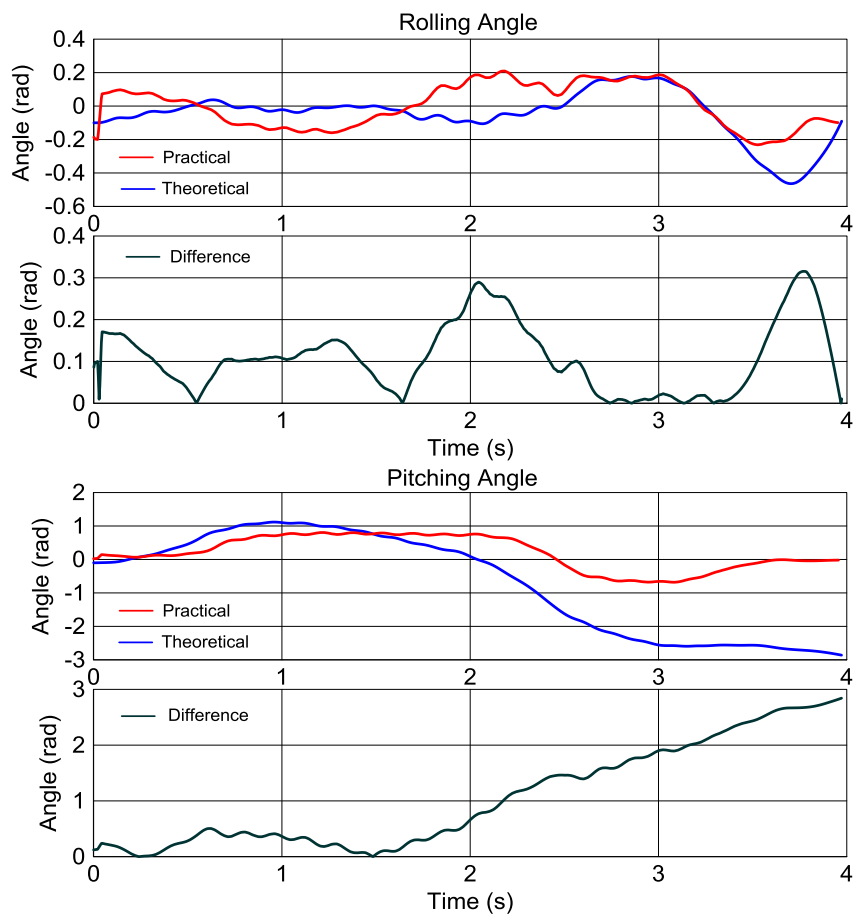

Fig. 3. Divergence of the practical and theoretical model. pitch was caused by the wind, since the wind acted on the front of the quadcopter for the duration of the flight. Thus it influenced the forwards and backwards pitching motion differently and can be considered as a cause of the slow decrease of the theoretical pitch angle from $t=1 \mathrm{sec}$ to $t=2.2 \mathrm{sec}$ and the overshoot at $t=2.5 \mathrm{sec}$, after which the theoretical UAV is not able to return to a horizontal position.

The inclusion of blade flapping, as considered in [14], [16], [19], [20] and of the effects of wind and drag into the model would have increased its accuracy. However, with the given data and project time constraints this was not possible. Hence, the mathematical model is considered limited in its current form, and not reliable for the precise prediction of the attitude of a UAV. Although, it is suitable to predict the general behaviour of a UAV configuration in a neutral environment such as those encountered during indoor flights or theoretical computer simulation and design environments.

\section{SIMULATION RESUlTS}

The theoretical UAV, which has been used for the simulation, has the properties given in Table 1.

\section{A. Yawing}

The main difference expected between the V-tail Quadcopter and a normal Quadcopter is the yawing rate. For these simulations the properties of Table 1 are used, with the exception that the weight is $1.5 \mathrm{~kg}$ on the V-tail configuration. During the simulation the copter was slowly flown downwards and after one second the rotational speed of motor 1 and 2 was increased and of motor 3 and 4 decreased by $1000 \mathrm{rpm}$ for $0.06 \mathrm{sec}$. To compensate the thrust reduction of a $\mathrm{V}$-tail, the rotational speed of the rear motors (M2, M4) was further increased by $360 \mathrm{rpm}$.

Fig. 4 shows the results of the simulation. As expected, the UAV with the dihedral angles has a slightly lower yawing rate, than the Normal Quadcopter, which is caused by the angled motors and the resulting splitting of $T_{q}(13)$ into the three axis of the coordinate system. The other components of this moment are cancelling each other out, just like the forces caused by the dihedral angles and therefore the UAV maintains the angular position around its other axes.

The V-tail configuration on the contrary is an asymmetrical configuration and needs different rotational speeds of the rear and front motors to maintain level flight. After the Yawing operation of the motors, a drift around the

TABle 1 Properties of The Theoretical Quadcopter

\begin{tabular}{|l|l|}
\hline Parameter & Value \\
\hline Mass & $1.2 \mathrm{~kg}$ \\
\hline Body inertia & {$[0.0125 ; 0.0125 ; 0.0215] \mathrm{kg} \cdot \mathrm{m}^{2}$} \\
\hline Motor and propeller inertia & $0.00004 \mathrm{~kg} \cdot \mathrm{m}^{2}$ \\
\hline Arm length $(x$ and $y)$ & $0.2 \mathrm{~m}$ \\
\hline Motor angles & $0 / 14 / 30^{\circ}$ \\
\hline Density of air & $1.225 \mathrm{~kg} / \mathrm{m}^{3}$ \\
\hline Gravity & $9.81 \mathrm{~m} / \mathrm{s}^{2}$ \\
\hline Propeller & $\mathrm{APC} 9 \times 4.5 \mathrm{MR} ;$ APC9x4.5MRP \\
\hline
\end{tabular}




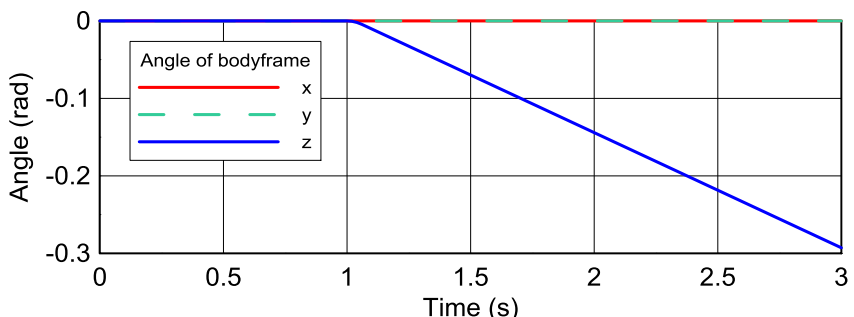

a)

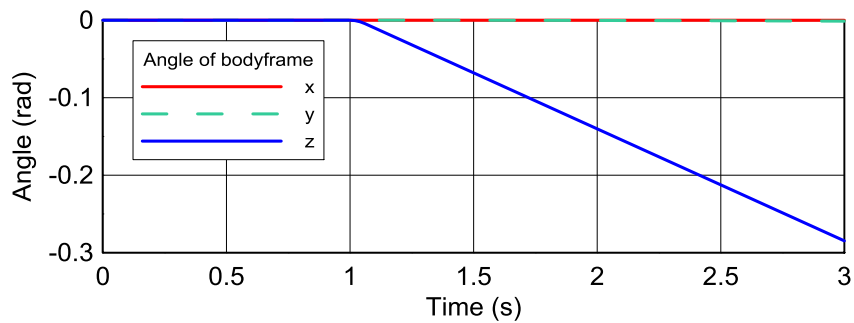

b)

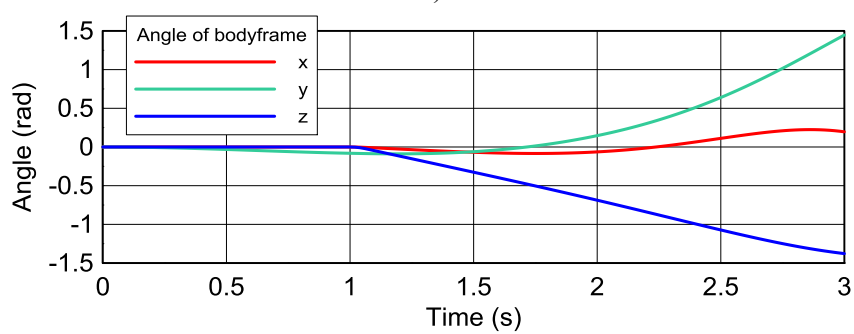

c)

Fig. 4. Angles of the UAV during the yawing simulation: (a) normal quad, (b) dihedral, (c) V-tail configuration.

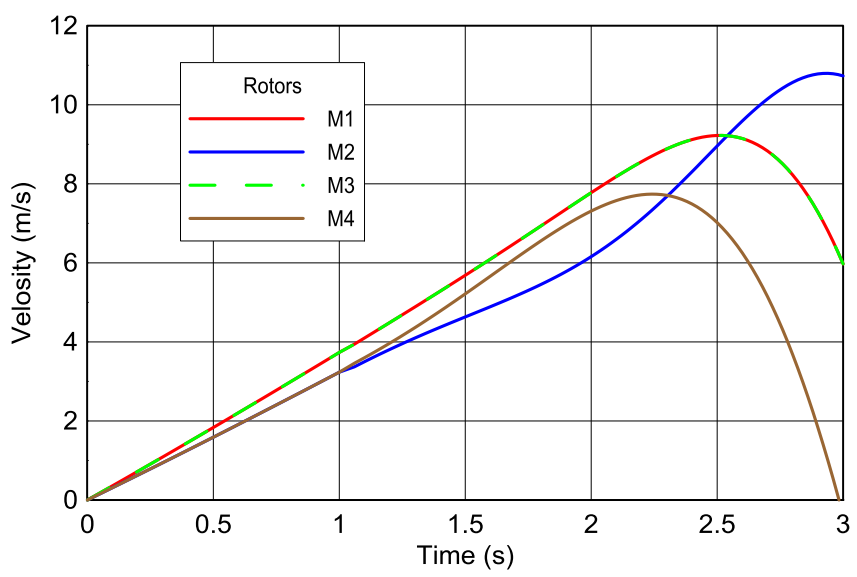

Fig. 5. Different velocities of the airstream normal to the propeller disc (Vtail configuration).

$X_{b}$ axis can be observed, which is caused by a different increase in thrust, since thrust is acting on M2 also along the $Y_{b}$ axis, which is causing a lower rate of thrust increase in $Z_{b}$ than that produced by $\mathrm{M} 1$. This $Y_{b}$ component is responsible for the significant higher Yawing rate of the $\mathrm{V}$ tail configuration. If no countermeasures are taken, the copter will oscillate around the $X_{b}$ axis, because of the sideway motion and the different velocities normal to the propeller disc of the rear motor (Fig. 5).

The lack of any control circuit in the simulation is the reason of the decrease of the angle around $Y_{b}$. The increase from $t=1 \mathrm{sec}$. can be explained with the different velocity of the airstream normal to the propeller disk (Fig. 5) and the resulting change of lift, since the thrust is reduced by approximately the same amount, but due to the tilting angle, the rear motors are not as efficient as the front ones.

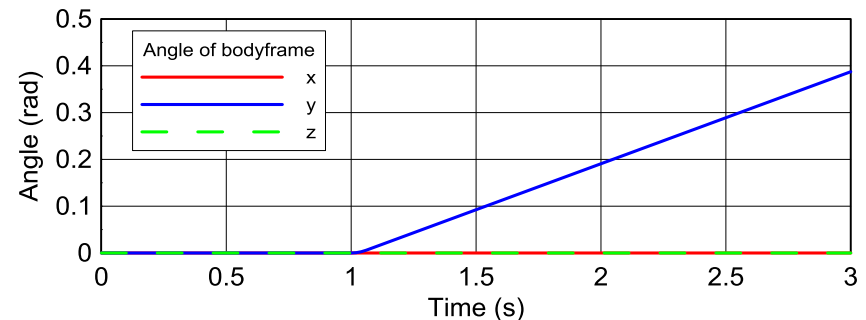

a)

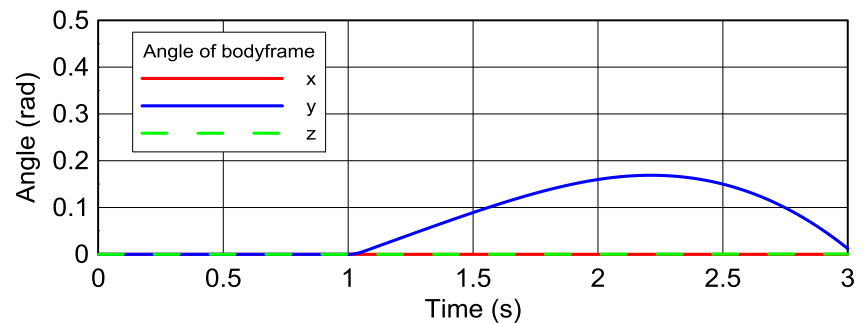

b)

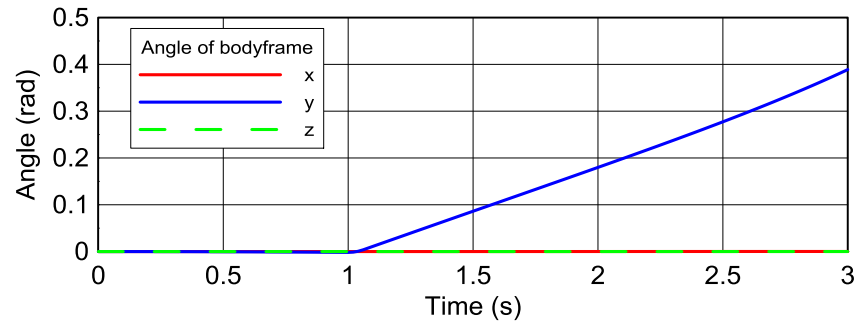

c)

Fig. 6. Angles of the UAV during the pitching simulation (a) normal quad, (b) dihedral, (c) V-tail configuration.

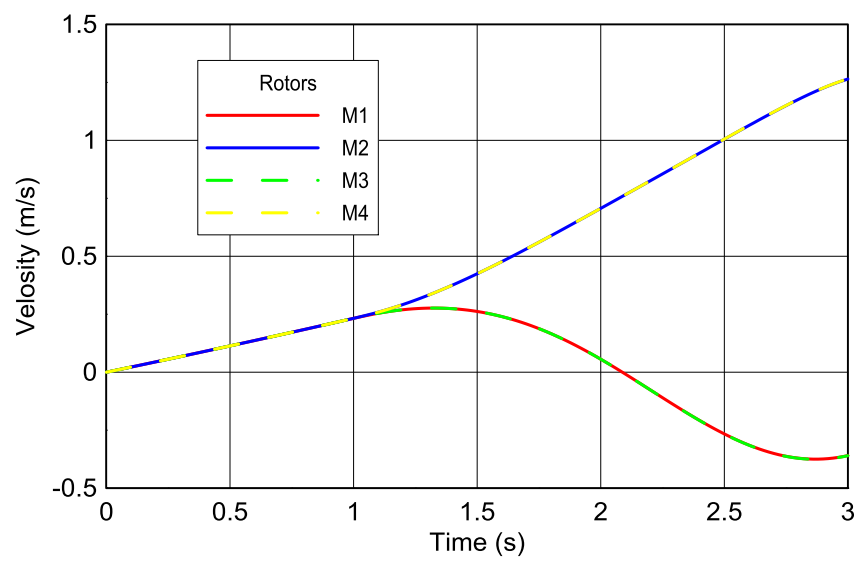

Fig. 7. Velocity normal to propeller disk, dihedral configuration. The initial velocity is caused by a slow ascend.

The result is a faster decrease of the lift on M2 and M4 than on M1 and M3 and a decrease of the UAVs pitch angle.

\section{B. Pitching}

To avoid the influences of the linear velocity along the $Z_{n}$ axis, which occurred during the yawing simulation with the "V-tail" configuration, the UAV has to maintain its height more precisely. This is achieved with a rotational speed of $6720 \mathrm{rpm}$, were the UAV is rising for about $7 \mathrm{~mm}$ in the first second. To compensate the "V-tail", the rotational speed of rear motors (M2, M4) on this configuration is increased by $382 \mathrm{rpm}$. After $t=1 \mathrm{sec}$. the rotational speed of the rear motors (M2, M4) is increased by $50 \mathrm{rpm}$ and of the front motors (M1, M3) decreased by $50 \mathrm{rpm}$ for $0.06 \mathrm{sec}$ (Fig. 6). For this simulation the properties of Table 1 are used. 
As expected, the Quadcopter without tilted motors has the fastest Pitching rate (Fig. 6). The minor difference between pitching rate of the "V-tail" and the "Normal" Quadcopter should be more significant at first sight. But since a component of $T_{q}$ is acting along the $Y_{b}$ axis, described in the previous chapter, the increase of the thrust of the rear motors is inducing a counter moment of the propeller, supporting the pitching. The components of these moments around $Z_{b}$ are cancelling each other out, but due to the different rotational directions and angles the moments around $Y_{b}$ are supporting each other. If the rotational directions of all motors are reversed, this effect would have negative effects on the pitching rate.

Special attention must be paid to the "Dihedral" configuration, where the angle decreases again, without any further change of the rotational speed of the motors. This is caused by the different velocities normal to the propeller disk, which is shown in Fig. 7. When the movement along $X_{b}$ starts, $\mathrm{XN}$ of $\mathrm{M} 1$ and M3 is decreasing due to the dihedral angles. $\mathrm{XN}$ of M2 and M4 is increasing because they are tilted towards the direction of the movement. These different velocities are causing different thrust coefficient (ct) values for each motor, therefore the rear motors are producing less thrust than the front ones.

For further examination of the Dihedral effect an angular disturbance around the $X_{b}$ axis of $0.1 \mathrm{Nm}$ for $0.016 \mathrm{sec}$ at $t=0.5 \mathrm{sec}$. is induced (Fig. 7). The same behaviour as in Fig. 5 can be noted, but the longer simulation time reveals that this behaviour multiplies until the angle and the angular velocity overcomes the thrust difference of the motors. A simulation with a constant $\bar{F}_{\text {disturbance }}$ along the $X_{b}$ axis was also performed and the same effect took place, since it is caused by the velocity of the UAV and not the tilting angle.

\section{PITCHING/ROLLING COMPARISON OF THE V-TAIL}

Since the "normal" and the "dihedral" configurations are symmetrical no rolling simulation was conducted, because it was assumed that their behaviour in these manoeuvres would be identical. The "V-tail" configuration on the other hand is unsymmetrical and will behave differently.

For this simulation the properties of Table 1 are used, but the rotational speed of the front motors were set to $7320 \mathrm{rpm}$ and the rear ones were set to $7712 \mathrm{rpm}$. The roll/ pitch manoeuvre was initialised by increasing the rotational speed of M2, M3 for rolling and M2, M4 for pitching, by $50 \mathrm{rpm}$. The rotational speed of the motors on the opposite side was decreased by $50 \mathrm{rpm}$, at the same time. This altering of the rotational speed took place at $t=1 \mathrm{sec}$ for $0.06 \mathrm{sec}$.

In Fig. 8 can be seen, that the rolling rate is slightly higher than the pitching rate. It is assumed, that this is caused by the front motor, which was not tilted and used all of the thrust to generate the moment.

During rolling the UAV is tilting around every axis, due to its unsymmetrical nature. To counter this, more complex controlling mechanisms are needed. It can be seen that the effect of the dihedral configuration occurs during rolling, because the velocity normal to the propeller disk is not the same during sideways movements (Fig. 9). Due to the characteristic shape of the airframe structure, this effect

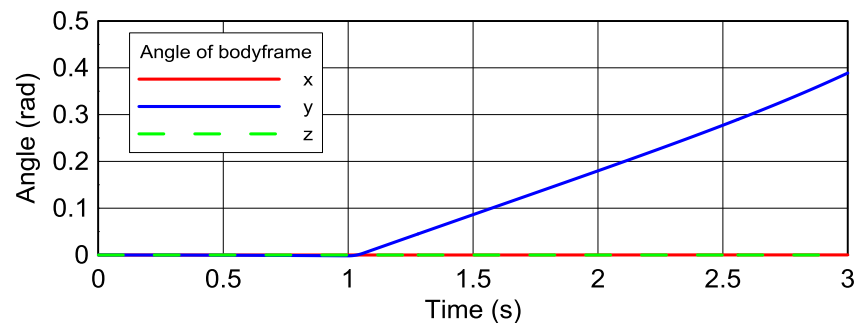

a)

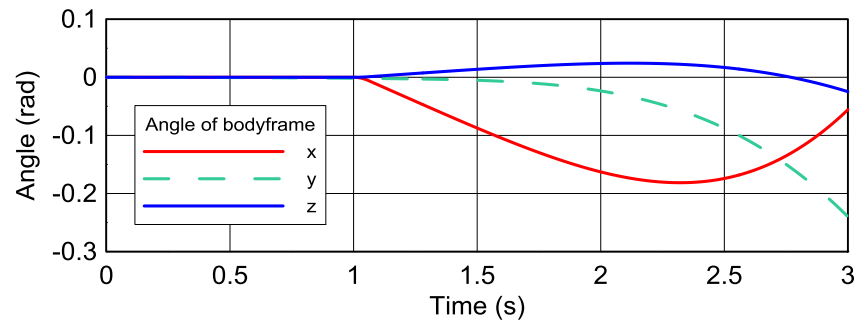

b)

Fig. 8. Roll (a) and pitch (b) of the V-tail configuration.

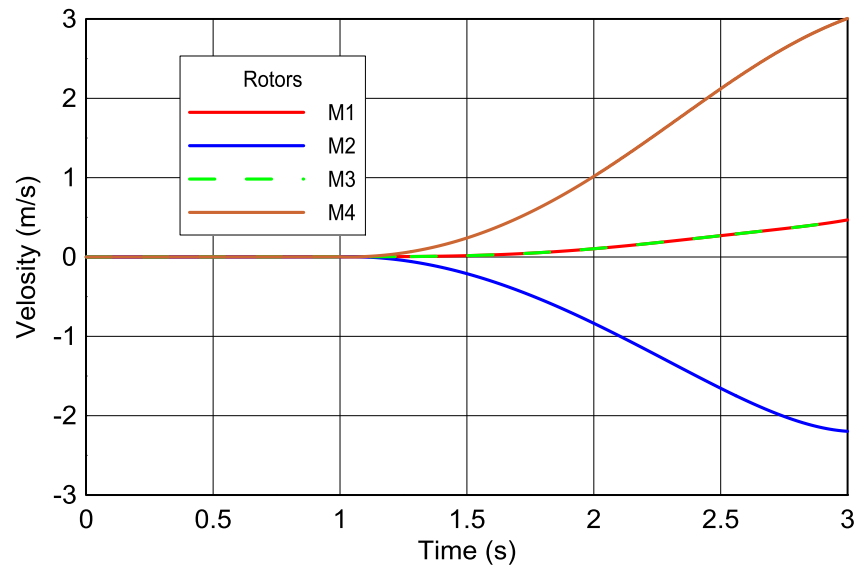

Fig. 9. Velocity normal to propeller disk, roll, V-tail.

occurs just on the rear motors, which is intensifying the yawing induced by the $Y_{b}$ component of the thrust.

\section{CONCLUSION}

In this paper, the author shows that, as expected, the most efficient quadcopter configuration is the "normal" one because all of the thrust is used in every manoeuvre and nothing is wasted. The "dihedral" configuration causes the Quadcopter to tilt against the direction of the movement if no countermeasures are taken. Which is caused by the different thrust coefficients of the motors. As stated in [12], this effect is contributing to the stability of a multirotor aircraft.

The yawing rate of the "V-tail" is significantly higher $(470 \%)$ than that of the "normal" configuration and the pitching rate is higher than expected, due to a component of the moment caused by the drag of the tilted propellers. Thus it is very important to consider the rotational direction of the motors otherwise this moment could make the Quadcopter less efficient. Another issue is that this configuration needs a special control circuit, due to the unsymmetrical thrust.

To support the simulation, test flights were performed, which were simulated. The attitudes of the real and the theoretical flight were compared and the discrepancies could be explained by the absence of wind, drag and blade 
flapping in the model. However, the model was considered to be accurate enough for the simulations which were performed.

\section{REFERENCES}

[1] E. Human, T. Harold, and C.W. Peter, "e-Reconstruction and earchiving of iconic architectural heritage: A complete example," in Proc. 22nd Int. Conf. Virtual System and Multimedia (VSMM), 17-21 Oct. 2016, Kuala Lumpur, Malaysia, pp. 1-8.

[2] D. Meyer, M. Hess, E. Lo, C.E. Wittich, T.C. Hutchinson, and F. Kuester, "UAV-based post disaster assessment of cultural heritage sites following the 2014 South Napa Earthquake," in Proc. Digital Heritage Int. Congress, 28 Sept.-2 Oct. 2015, Granada, Spain, pp. 421-424.

[3] H. Gonzalez-Jorge, J. Martinez-Sanchez, M. Bueno, and P. Arias, "Unmanned aerial systems for civil applications: A review", Drones, vol. 1, no. 1, pp. 1-19, 2017.

[4] L. Garcia-Delgado, A. Dzul, and V. Santibanez, "Quad-rotors formation based on potential functions with obstacle avoidance," IET Control Theory Applications, vol. 6, no. 12, pp. 1787-1802, 2012.

[5] P. Vlantis, P. Marantos, C.P. Bechlioulis, and K.J. Kyriakopoulos, "Quadrotor landing on an inclined platform of a moving ground vehicle," in Proc. IEEE Int. Conf. Robotics and Automation, 26-30 May 2015, Seattle, USA, pp. 2201-2207.

[6] M. Bergamasco, and M. Lovera, "Identification of linear models for the dynamics of a hovering quadrotor," IEEE Trans. Control Syst. Technol., vol. 22, no. 5, pp. 1696-1707, 2014.

[7] S. Omari, M.-D. Hua, G. Ducard, and T. Hamel, "Hardware and software architecture for nonlinear control of multirotor helicopters," IEEE/ASME Trans. Mechatronics, vol. 18, no. 6, pp. 1724-1736, 2013.

[8] S. Mintchev, S. de Rivaz, and D. Floreano, "Insect-inspired mechanical resilience for multicopters," IEEE Robotics Automation Letters, vol. 2, no. 3, pp. 1248-1255, 2017.

[9] F. Chui, G. Dicker, and I. Sharf, "Dynamics of a quadrotor undergoing impact with a wall," in Proc. Int. Conf. Unmanned Aircraft Systems (ICUAS), 7-10 June 2016, Arlington, USA, pp. 717 $-726$.
[10] J. Toledo, L. Acosta, D. Perea, and N. Morales, "Stability and performance analysis of unmanned aerial vehicles: Quadrotor against hexrotor," IET Control Theory Applications, vol. 9, no. 8, pp. 11901196, 2015.

[11] H. Efraim, A. Shapiro, and W. Gera, "Quadrotor with a dihedral angle: on the effects of tilting the rotors inwards," Journal Intelligent Robotic Syst., vol. 80, no. 2, pp. 313-324, 2015.

[12] A.T. Ciarfuglia, A. Ficola, P. Valigi, and P. Valigi, "A preliminary experimental analysis of V-tail quad-rotor dynamics," in Proc. 6th Int. Conf. Modelling, Identification and Control (ICMIC), 3-5 Dec. 2014, Melbourne, Australia, pp. 277-282.

[13] D.K. Sebesta, and N. Boizot, "A real-time adaptive high-gain EKF, applied to a quadcopter inertial navigation system," IEEE Trans. Industrial Electronics, vol. 61, no. 1, pp. 495-503, 2014.

[14] R. Mahony, V. Kumar, and P. Corke, "Multirotor aerial vehicles: Modeling, estimation, and control of quadrotor," IEEE Robotics Automation Magazine, vol. 19, no. 3, pp. 20-32, 2012

[15] J. Brandt, and M. Selig, (2011, Jan 4) UIUC propeller data site. [Online]. Available: http://m-selig.ae.illinois.edu/props/propDB.html

[16] E. Davis, and P.E.I. Pounds, "Direct sensing of thrust and velocity for a quadrotor rotor array," IEEE Robotics Automation Letters, vol. 2, no. 3, pp. 1360-1366, 2017.

[17] P. Castillo, A. Dzul, and R. Lozano, "Real-time stabilisation and tracking of a four-rotor mini rotorcraft," IEEE Trans. Control Syst. Technol., vol. 12, no. 4, pp. 510-516, 2014.

[18] B. Earl, (2016, May 9) Adafruit. [Online]. Available: https:// learn.adafruit.com/adafruit-data-logger-shield/using-the-real-timeclock-3

[19] R.W. Deters, G.K.A. Krishnan, and M.S. Selig, "Reynolds number effects on the performance of small-scale propellers," in Proc. 32nd AIAA Applied Aerodynamics Conf., June 2014, Atlanta, USA, pp. 148.

[20] J.B. Brandt, and M.S. Seilig, "Propeller performance data at low Reynolds numbers," in Proc. 49th AIAA Airospace Science Meeting, 4-7 Jan. 2011, Orlando, USA, pp. 1-18. 